\title{
Accountability y sociedad civil: el control político en la era digital*
}

\author{
Accountability and Civil Society: Political \\ Control in the Digital Era
}

\author{
Alejandra Ríos Ramírez** \\ Juan Esteban Garro***
}

Recibido: 30 de septiembre de 2016

Aprobado: 1 de diciembre 2016

Publicado en línea: 10 de octubre de 2017

\section{Resumen}

Este artículo tiene como objetivo exponer el peso que tienen actualmente las tecnologías de la información y la comunicación en el ejercicio del accountability societal. Para ello, se hace una reconstrucción del término accountability teniendo en cuenta sus dimensiones y tipologías. A continuación, se hace un contraste entre gobierno electrónico, gobierno abierto y el discurso de la transparencia. Concluimos que, aunque el análisis de los discursos y las propuestas que buscan ligar el uso de herramientas digitales a la promoción de la transparencia y el control político permite identificar avances significativos en lo concerniente al desarrollo de aplicaciones y portales para la liberación de datos estatales,

\section{Abstract}

This article aims to expose the weight that information and communication technologies currently have on the exercise of societal accountability. For this, a reconstruction of the term accountability is made, taking into account its dimensions and typologies. Next, a contrast is made between electronic government, open government and the discourse of transparency. We conclude that, although the analysis of the discourses and proposals that seek to link the use of digital tools for the promotion of transparency and political control allows to identify significant advances in the development of applications and portals for the release of state data, the distinction between

\footnotetext{
* Este artículo es producto de la investigación realizada en el proyecto Rendición de cuentas: mecanismo democrático para el control político, que hace parte de la convocatoria nacional para formación de jóvenes investigadores Colciencias n. ${ }^{\circ} 617$ de 2013. Artículo de investigación

** Filósofa y magíster en Filosofía por la Universidad de Antioquia. Profesora de tiempo completo del Departamento de Gobierno y Ciencias Políticas de la Universidad Eafit y directora del Observatorio Parlamentario Antioquia Visible adscrito al Centro de Análisis Político del mismo departamento. Correo electrónico: ariosram@eafit.edu.co *** Psicólogo por la Universidad de Antioquia, politólogo por la Universidad Eafit y maestrando en Comunicación y Política de la Universidad Autónoma Metropolitana de la Ciudad de México. Profesor de la Universidad Eafit. Correo electrónico: jgarrove@eafit.edu.co ORCID: http://orcid.org/0000-0002-9050-4098
} 
no es muy clara la distinción entre las apuestas por la circulación libre de la información y las posibilidades reales de sanción y control sobre el Estado que aquellas promueven. Hemos hallado, mediante una exhaustiva revisión bibliográfica, que esa falta de claridad es un obstáculo para para conciliar el anhelo de gobiernos abiertos y transparentes con herramientas de fiscalización, monitoreo y sanción a la conducta de representantes y funcionarios públicos. A pesar de lo anterior, proponemos que dirigir la mirada de manera seria a las plataformas móviles como herramientas de control político posibilitaría una suerte de "gobernanza móvil" a partir de la cual se susciten prácticas de gobierno abierto articuladas a la real posibilidad de que los ciudadanos activos movilicen las agencias estatales orientadas a la sanción de aquellos funcionarios públicos que se excedan en sus ejercicios de administración y poder político.

\section{Palabras clave}

accountability; internet; gobierno abierto; sociedad civil; control social

\section{Cómo citar este artículo:}

Ríos-Ramírez, A. y Garro, J. E. (2017). Accountability y sociedad civil: el control político en la era digital. Papel Político, 22(2), 311-337. https://doi.org/10.11144/Javeriana. papo22-2.ascc bets for the free circulation of information and the real possibilities of sanction and control over the State that they promote is not very clear. We have found, through an exhaustive literature review, that this lack of clarity is an obstacle to reconcile the longing for open and transparent governments with tools for inspection, monitoring, and sanctioning the behavior of representatives and public officials. In spite of the above, we propose that taking a serious look at mobile platforms as tools of political control, would allow a kind of "mobile governance" from which open government practices are articulated to the real possibility that active citizens mobilize state agencies aimed at sanctioning those public officials who exceed their administrative and political power exercises.

\section{Keywords:}

accountability; internet; open government; civil society; social control 


\section{Introducción}

Aunque la expresión más básica de un régimen político democrático es que sus procesos electorales son periódicos, libres y competitivos, también es cierto que la dimensión representativa de la democracia es insuficiente. Esto es, si la legitimación de un sistema político pasa solo por el ejercicio de la participación electoral, el Estado puede reducirse a mero instrumento de validación, mediación y gestión de las preferencias de las mayorías. El derecho de elegir a quienes nos gobiernan y permitir que esos actores vehiculen nuestras demandas conlleva un deber igual de importante que el derecho de elección: la constante vigilancia y el monitoreo de las decisiones y acciones de los funcionarios públicos. Así pues, la participación política, entendida también como control al poder público, es uno de los elementos fundamentales de las democracias contemporáneas. Tal control, conocido desde la literatura politológica como accountability, permite ampliar el sentido de la participación desde una perspectiva cívica y, por lo mismo, más comprometida con los asuntos que a todos nos conciernen como ciudadanos. De acuerdo con lo anterior, este artículo pretende exponer el peso que en la actualidad las tecnologías de la información y la comunicación (TIC) tienen para el ejercicio del accountability societal. Para lograr lo anterior, se hace una reconstrucción del término accountability, teniendo en cuenta sus dimensiones y tipologías. A continuación, se hace un contraste entre gobierno electrónico, gobierno abierto y el discurso de la transparencia. Relacionando el accountability con las categorías anteriores, se presentan algunas críticas a la idea del control político desde la perspectiva del gobierno abierto, pero también se proponen alternativas para fortalecer las posibilidades de participación ciudadana, de supervisión y monitoreo a la función estatal desde la sociedad civil en esta era digital.

\section{Accountability: una caracterización general}

Schedler afirma que el accountability, en cuanto término en boga (fashionable term), puede entenderse como aquel que "expresa la demanda continua de revisión y supervisión, de vigilancia y constreñimientos institucionales al ejercicio del poder” (1999, p. 13). La inquietud de Schedler por el control a la praxis política no es nueva, sino que esta puede rastrearse, incluso, en la antigua Grecia. ${ }^{2}$ Controlar el ejercicio del poder público implica evitar que aquellos que lo poseen incurran en excesos en su administración y así garantizar la satisfacción de las necesidades ciudadanas y la

\footnotetext{
${ }^{1}$ Es importante aclarar que en ciencia política no existe una definición unívoca del término. Sin embargo, para desarrollar este apartado, se toman apuntes y definiciones de teóricos ampliamente reconocidos en el campo que coinciden en cuanto a presupuestos semánticos y prácticos.

${ }^{2}$ Atendiendo a términos estrictos desde la ciencia política, no es preciso hablar de accountability en la antigua Grecia. Sin embargo, es posible rastrear ejercicios de rendición de cuentas y apuestas institucionales y formales para regular el poder. Este artículo apela de manera indistinta tanto a rendición de cuentas como a accountability. Sin embargo, para ampliar la noción a propósito de la evolución del término y su diferencia con la rendición de cuentas, puede revisarse Elster (1999), O'Donnell (1998, 2001, 2004), Bovens (2007), Ríos, Cortés, Fuentes y Suárez (2014).
} 
protección de los derechos civiles. Sin embargo, este ideal se enfrenta a una realidad compleja: constreñir la capacidad ejecutoria de administradores públicos puede limitar en muchos sentidos su margen de acción (Dunn, 1999; O’Donnell, 2004). Esta ambigüedad da lugar a una de las características fundantes del accountability: la armonía entre una gestión estatal eficiente y eficaz y la observancia de normas que buscan ajustar dicha gestión a principios ético-públicos de conducta de los funcionarios (O’Donnell, 1998, 2004).

La fórmula para que el control a las acciones estatales pueda armonizarse con las funciones de representantes y funcionarios públicos se encuentra en las dos dimensiones constitutivas del accountability, identificadas por Schedler $(1999,2008)$ como answerability y enforcement.

El answerability resalta la importancia de la transparencia de cualquier acto estatal frente a la ciudadanía y del debate ampliado para la toma de decisiones y ejecución de acciones de gobierno, por lo cual hace referencia tanto a "la dimensión informativa informar, dar datos, narrar en público- como a la dimensión argumentativa -explicar, dialogar, escuchar- de la rendición de cuentas, y presupone obligación y responsabilidad de los funcionarios de acatar y ejercer estas dos funciones" (Hernández y Arciniegas, 2011, p. 25). De acuerdo con lo anterior, los funcionarios deben informar sobre sus actuaciones y decisiones y, asimismo, estar prestos a responder a los señalamientos o interrogantes que su actuar suscite.

Por lo anterior, es preciso que la sociedad civil disponga de las herramientas jurídicas y políticas necesarias para exigir ajuste de cuentas y explicaciones sobre la conducta de quienes los representan (Sosa, 2011). No obstante, asegurar el answerability permite identificar solo un control político nominal. Debe existir también la posibilidad de sanciones reales a disfuncionalidades que puedan derivarse del ejercicio del poder público. Posibilidad que configura, según Schedler (2008), la segunda dimensión del accountability: el enforcement.

Argumenta Bovens (2007) que no es necesario que la sanción se haga efectiva para que haya accountability, sino que la factibilidad del castigo sería un estímulo suficiente para disuadir a funcionarios públicos de comportamientos que se alejen de los preceptos normativos que los rigen. Solo pueden ser identificados ejercicios reales de accountability cuando se constata la presencia de ambas dimensiones (Sosa, 2011; Fox, 2007).

La caracterización general que este apartado propone exige, además, ocuparse de la tipología del accountability. En primer lugar, puede reseñarse el accountability horizontal. Desde su perspectiva, el Estado es visto como un sistema complejo, integrado por diferentes componentes y estructuras que al articularse permiten establecer el diálogo necesario para el planteamiento, la aprobación y la ejecución de decisiones públicas. Articulación que a la par facilita la existencia de un régimen de fiscalización y control en 
el Estado (O’Donnell, 2004). Ello gracias a la presencia de agencias que cuentan con las competencias, capacidades y herramientas legales para activar mecanismos de supervisión a las acciones de las demás dependencias estatales. Agencias que pueden ser de dos tipos: las primeras son las dependencias regulares del Estado que, además de buscar articularse para tomar decisiones de gobierno, se constituyen en agentes supervisores del equilibrio de poderes en el Estado. Estas dependencias activan el accountability horizontal de balance;

sin embargo, las agencias que lo activan no tienen como función principal la de supervisar e inspeccionar las actuaciones de los demás agentes estatales (O'Donnell, 2004; Hernández y Arciniegas, 2011). Por consiguiente, para optimizar y garantizar el cumplimiento de los ejercicios de vigilancia, se establecen las agencias de accountability horizontal asignado destinadas estrictamente a la fiscalización y el escrutinio de cada una de las actividades público-administrativas que tienen lugar en el Estado. Por esta razón, es preciso que ellas sean independientes del órgano ejecutivo (O’Donnell, 2004; Peruzzotti, 2008, 2010; Kenney, 2000).

Las agencias de accountability horizontal asignado son "un útil complemento y refuerzo de las instituciones de balance" (O’Donnell, 2004, p. 22). No obstante, desde la perspectiva del accountability desarrollada por el mismo O'Donnell, resultan insuficientes. El carácter representativo de las democracias liberales contemporáneas exige un mínimo de participación civil, materializado, inicialmente, en la delegación del poder que se produce en el momento en que los ciudadanos votan por sus representantes. Las elecciones democráticas permiten identificar el segundo tipo de accountability en la tipología diseñada por O’Donnell (1998, 2004): accountability vertical electoral. Se diferencia del accountability horizontal, porque alude a un ejercicio exterior de control sobre el aparataje estatal, que asciende desde la base de la sociedad hacia el Estado.

Ahora bien, este mecanismo electoral ofrece sustanciales limitaciones a la posibilidad de control y supervisión a los entes gubernamentales desde la sociedad civil. En términos prospectivos, no hay forma de asegurar la concreción de la agenda programática que ha llevado a la elección de un grupo particular de candidatos. Es posible señalar un vínculo deficitario propio de las elecciones como herramienta para el control civil, en la medida en que los ciudadanos no cuentan con las fuentes de información suficientes y cualificadas para hacer un seguimiento transversal en el tiempo a la acción de sus representantes, quienes cuentan con un amplio margen de discrecionalidad entre los periodos electorales. A ello se suman la corrupción y el clientelismo tanto endógenos como exógenos y el hecho de que no todos los cargos públicos puedan ser reelegidos. Más aún, muchos de los funcionarios responsables de la ejecución directa de las diferentes políticas públicas se deben contractualmente a la figura de libre nombramiento y remoción. De igual forma, es difícil pensar que el voto pueda ser el único mecanismo 
con que cuentan los ciudadanos para regular acciones estatales que pueden afectar múltiples capas del entramado social. ${ }^{3}$

\section{Accountability social: la domesticación del poder político desde una perspectiva ampliada}

Las críticas señaladas permiten afirmar que es necesario considerar perspectivas sociales de control complementarias a las que supone el mecanismo del voto y que hacen parte de una categoría conocida como accountability social o societal. De acuerdo con Peruzzotti y Smulovitz, "son un tipo de rendición de cuentas no electoral de las autoridades políticas basado en las acciones de un amplio espectro de asociaciones y movimientos ciudadanos" (2002, p. 10); su valor radica en que acercan a la sociedad civil a las cuestiones políticas más inmediatas (O’Donnell, 2004; Peruzzotti, 2008). ${ }^{4}$ El accountability social reconoce, además, que la manera en que la sociedad civil se agremia y manifiesta, formal o informalmente, desempeña un papel fundamental en los procesos de reconfiguración y cambio de las estructuras de gobierno (Fox, 2000).

Los movimientos sociales, las ONG, los centros de pensamiento y los medios de comunicación independientes son los actores que encarnan y escenifican los presupuestos del accountability social (Peruzzotti, 2010). Fundan su actividad en el derecho que emana de la sociedad civil para señalar deficiencias en el desempeño estatal y así activan agencias de control horizontal (accountability horizontal de balance y asignado) responsables de vigilar y sancionar el comportamiento de políticos y funcionarios públicos. ${ }^{5}$

Los movimientos sociales son, desde la tipología descrita por Peruzzotti (2010), el primer actor del accountability social. Una de sus características básicas es que son producto, muchas veces, de factores coyunturales en los que la sociedad civil termina afectada debido a acciones u omisiones por parte de agentes públicos. Los movimientos sociales, formales e informales, ${ }^{6}$ elevan demandas con movilizaciones y

\footnotetext{
${ }^{3}$ Para ampliar la información a propósito de las críticas hechas al accountability vertical electoral, puede verse Hernández y Flórez (2011), Peruzzotti y Smulovitz (2002), Peruzzotti (2008, 2010, 2012), Fox (2007), Montoya (2010) y O'Donnell $(1998,2004)$.

${ }^{4}$ Es importante advertir que, desde la literatura sobre accountability, el despliegue de estas acciones de intervención de la sociedad sobre el Estado se ha enmarcado a lo largo de los últimos veinte años en tres estrategias principales: jurídicas, de movilización y mediáticas (Peruzzotti y Smulovitz, 2002; O’Donnell 2004; Fox, 2007; Schedler, 2008; Peruzzotti, 2010; Hernández y Flórez, 2011; Ríos y Trujillo, 2014). Este artículo se ocupará en mayor medida de las dos últimas estrategias, esto es, las de movilización y las mediáticas.

${ }^{5}$ En la misma línea de las manifestaciones sociales del accountability, es válido hacer una diferenciación. Aunque las manifestaciones del accountability social emergen desde la base misma de la sociedad, debe distinguirse las iniciativas propias de actores colectivos o sociales de las impulsadas por actores individuales respecto de las demandas personales al Estado vía tutelas, acciones populares, acciones de cumplimiento y denuncias (Hernández y Flórez, 2011, p. 25).

${ }^{6}$ Pueden encontrarse diferencias sustanciales entre agremiaciones sociales que trabajan para fines específicos independiente de las coyunturas económicas y políticas de aquellas que resultan motivadas por hechos sociales o políticos concretos. Para más información sobre estas diferencias y la naturaleza misma de los movimientos sociales en el accountability social, puede consultarse Ríos y Trujillo (2014).
} 
manifestaciones que permiten señalar déficits en los servicios prestados por el Estado y exigir claridad y justificaciones frente a la actuación de algún funcionario o sanciones por desbordes en la administración del poder público.

El segundo actor del accountability social, según Peruzzotti (2010), está compuesto por las ONG, las organizaciones de la sociedad civil y los centros de pensamiento. Los grupos que encarnan este tipo de "asociativismo cívico" reflejan altos niveles de formación profesional y académica y de autonomía e independencia frente a entidades gubernamentales en relación con las acciones que despliegan (Ríos y Trujillo, 2014). La autonomía, y su formación y recorrido profesional, permiten describirlas como organizaciones sociales de élite con gran capacidad de recolección, sistematización y análisis de información sobre amplios ejes de intervención estatal. A diferencia de muchos de los movimientos sociales, estas organizaciones no son de naturaleza coyuntural, por lo cual logran ocuparse, merced a su presencia permanente y de largo alcance, del análisis transversal de problemas en la arquitectura y el funcionamiento del Estado (Peruzzotti, 2010; Ríos y Trujillo, 2014). De esta forma, el "asociativismo cívico" termina constituyéndose en recurso para alimentar las demás tipologías del accountability, porque genera análisis, insumos teóricos y técnicos e indagaciones prácticas y empíricas que servirán para sustentar las acciones de control y vigilancia que, eventualmente, deseen emprender agencias asignadas o de balance.

En tercer lugar, aparecen los medios de comunicación y el periodismo de denuncia como agentes de accountability social. De acuerdo con Peruzzotti (2010), las acciones que ambos emprenden tienen un carácter dual: por un lado, son claves en la difusión y visibilización de las demandas hechas por movimientos sociales, las ONG, los centros de pensamiento o los grupos sociales que no cuentan con niveles de representación óptimos y cualificados en esferas institucionales o que han sido sistemáticamente relegados de las discusiones públicas. Por otro lado, sus indagaciones periodísticas e investigativas sirven como puente para divulgar a la ciudadanía y demás agentes del Estado las conductas corruptas, ineficientes e irregulares que puedan tener lugar en las instituciones públicas.?

Las perspectivas esbozadas por O’Donnell, Fox y Peruzzotti, a propósito de la intervención ciudadana en los procesos de control político, coinciden en que las sinergias entre los tres tipos de accountability - horizontal, vertical y social- no pueden

\footnotetext{
${ }^{7}$ Otra de las formas en que los medios de comunicación y el periodismo pueden constituir una fuente importante de accountability social tiene que ver con lo que ha convenido en llamarse la sociedad mediática. Según los análisis hechos por Thompson (2001), las formas en que la sociedad contemporánea se inclina a consumir escándalos políticos, así como a la rápida difusión de informes noticiosos (Castells, 2001, 2009), llevan a que puedan verse significativamente mermados los niveles de reputación y confianza con que podían contar los funcionarios públicos que llegaran a ser denunciados y veían menguado su capital social y político y, por ende, su poder simbólico, con lo cual se constituían, además, en el centro de atención e investigación de las agencias estatales que se ocupan de vigilar su gestión (Thompson, 2001).
} 
más que potenciar y cualificar las estrategias de vigilancia y supervisión existentes. El accountability puede identificarse como herramienta válida para el control político solo en la medida en que las acciones desarrolladas por cualquiera de los tres actores reseñados genere consecuencias políticas, sociales y legales factibles de ser identificadas (Peruzzotti y Smulovitz, 2002), esto es, activando agencias institucionales asignadas o de balance o influenciando la agenda política, mediática o jurídica de un país, al igual que mediante la sanción social y simbólica a funcionarios públicos que incurrieren en conductas que sobrepasen los linderos normativos fijados.

\section{Internet, plataformas mediáticas y accountability}

Internet inaugura un nuevo capítulo en las formas de gestión y administración pública. Las diferentes plataformas digitales y comunicacionales ${ }^{8}$ han sido propuestas desde finales de la primera década del siglo XXI como herramientas para nutrir los ejercicios de accountability y reforzar el vínculo entre el Estado y los ciudadanos en el seno de las democracias representativas actuales.

En uno de sus primeros actos como presidente de los Estados Unidos, Barack Obama abre la puerta a nuevas formas de control sobre las acciones estatales con el Memorandum on Transparenc y and Open Government del 21 de enero de 2009. Dos propósitos lo componen: 1) privilegiar como instrumento potenciador de la transparencia y el control gubernamental las TIC y 2) identificar estas herramientas como aquellas que permitirían establecer puentes directos de comunicación entre el Estado y la sociedad civil, con el fin de revitalizar la confianza ciudadana en los asuntos públicos, optimizar los distintos niveles administrativos para alcanzar mayor eficiencia y eficacia e impulsar la participación ciudadana.

El proyecto de apertura administrativa del gobierno propuesto por el Memorandum on Transparenc y and Open Government tiene por base tres presupuestos: ${ }^{9}$

- La transparencia: definida como el principio que debe inspirar a los Estados para dar cuenta, siempre y en todo lugar, de sus acciones, inversiones y gastos, planes en el futuro, fuentes de datos y su nivel de compromiso y responsabilidad frente a la sociedad.

\footnotetext{
${ }^{8}$ Es importante aclarar que por plataformas comunicacionales o mediáticas —como serán también nombradasse hace referencia a herramientas digitales que permiten la interacción entre los ciudadanos y el Estado, y que adoptan la forma de aplicaciones móviles, blogs, webs estatales o de organizaciones de la sociedad civil y sistemas de redes sociales.

${ }^{9}$ El ideal de Barack Obama, hecho discurso formal y categorizado en los tres principios enunciados, fue adoptado por muchas de las administraciones estatales en la búsqueda de un nivel de apertura sin precedentes en el Gobierno. La consecuencia más importante del memorando del presidente Obama en el mundo es la creación de la Alianza para el Gobierno Abierto (Open Government Partnership). Esta nace como iniciativa de ocho gobiernos: Brasil, Indonesia, México, Noruega, Filipinas, Sudáfrica, el Reino Unido y los Estados Unidos. En la actualidad, más de 64 países son miembros (https://www.opengovpartnership.org/).
} 
- La participación: se considera el principio que promulga e incentiva el derecho de los individuos a hacer parte activa en la identificación de los problemas que los afectan en el diseño de las soluciones sociales a estos problemas y en el seguimiento y la optimización a la implementación de estas con la creación de espacios de encuentro para la implicación ciudadana.

- La colaboración: es el principio con el que se busca que los espacios creados para la implicación y deliberación ciudadana se traduzcan en acciones conjuntas entre el Estado, la sociedad civil y la empresa privada, trascendiendo la etapa del diálogo para llegar a la de la acción colaborativa.

\section{Gobierno electrónico y gobierno abierto}

Pese a que el discurso del presidente Obama es visto en la literatura sobre transparencia y gobierno abierto como fundante de un nuevo paradigma en la gestión pública, pueden rastrearse antecedentes de la apuesta por vincular internet como herramienta para la administración del poder desde la última década del siglo XX. El uso de aplicaciones electrónicas con fines gubernamentales es identificado como e-government o gobierno electrónico, y ha sido adoptado por varios Estados alrededor del mundo desde principios de 1990. La Sociedad Americana de Administración Pública (ASPA 'American Society for Public Administration') define el gobierno electrónico como el uso innovador y práctico de las TIC e internet para la prestación eficiente y eficaz de servicios respecto de la información y el conocimiento. La implementación del gobierno electrónico tiene como fin fortalecer el compromiso de los representantes y funcionarios públicos con los ciudadanos y las organizaciones privadas y sociales.

Los defensores del gobierno electrónico veían en las herramientas brindadas por TIC una oportunidad para facilitar los flujos monetarios e informacionales alrededor del mundo, lo cual agilizaría las economías de escala y sistematizaría los servicios administrativos. Además, para ellos, el gobierno electrónico permitía crear nuevos recursos de producción, almacenamiento y circulación de los datos estatales, e incentivar los canales de diálogo con la sociedad impulsando la participación civil, los valores democráticos, la transparencia y el accountability (Dutton, 2009; Ahn y Bretschneider, 2011). De igual manera, los dispositivos electrónicos permitían que los servicios prestados por el Estado fueran más rápidos y sencillos, que se estimularan la eficiencia interna y la visibilidad de la gestión pública (Wong y Welch, 2004; Beckers, 2012).

Los análisis y reportes iniciales, elaborados a finales de la primera década del siglo XXI sobre los resultados de la implementación de un gobierno electrónico que era apenas incipiente (Ke y Wei, 2004; Lee, Tan y Trimi, 2005; Lee, 2008; Dutton, 2009; Ahn y Bretschneider, 2011) señalan el crecimiento de indicadores de eficiencia, disponibilidad y accesibilidad a servicios públicos y provisión de información relevante para la ciudadanía. 
Sin embargo, los análisis similares hechos desde otros sectores de la academia para finales del mismo periodo mantuvieron una posición escéptica frente a las grandes promesas del gobierno electrónico: no veían con claridad la mejora en los niveles de transparencia, accountability y confianza social en el Estado, ni podían identificar la transformación y optimización en la prestación de los servicios estatales que destacaban por ser sumamente restringidos y específicos (West, 2004; Ahn y Bretschneider, 2011). Finalmente, se cuestionaban las posibilidades reales de universalización de los ideales prometidos, ya que aparecían muchos límites para la facilidad en el uso de internet debido a los costos materiales y el nivel de experticia y conocimiento que implicaba para el usuario potencial.

La principal objeción hecha al gobierno electrónico tenía que ver con el pobre fortalecimiento del lazo entre la ciudadanía y el Estado. Los procesos de gobierno centrados en TIC para la prestación de servicios eran unidireccionales (Freeman, 2013). Esto es, las demandas elevadas desde la sociedad civil estaban restringidas a la calificación del servicio prestado o a la presentación de denuncias en línea, con lo que se acentuaba la visión del ciudadano como cliente o beneficiario final y espectador pasivo de lo que le ofrecía el aparato estatal (Ramírez-Alujas, 2012).

Los avances tecnológicos acaecidos a lo largo de los últimos diez años, conducentes a una arquitectura de la web mucho más óptima y eficiente, la globalización de las TIC, las críticas y limitaciones señaladas a propósito del gobierno electrónico y el Memorandum on Transparenc y and Open Government permiten el tránsito y la ampliación del gobierno electrónico a nuevos linderos fijados por la aparición del gobierno abierto (Wong y Welch, 2004; Ramírez-Alujas, 2012).

El gobierno abierto no parte solo del apoyo y respaldo ofrecido por apéndices tecnológicos, sino que, además, aspira a una transformación sustancial de la relación del Estado con la sociedad civil basada en la apertura estatal, mayor transparencia, participación y colaboración efectivas en escenarios que conjugan actores sociales, empresariales y gubernamentales. Así las cosas, la diferencia conceptual más importante entre el gobierno electrónico y el gobierno abierto $^{10}$ (Ahn y Bretschneider, 2011; Freeman, 2013) tiene que ver con que el segundo se vincula al desarrollo de aplicaciones destinadas a una comunicación, deliberación e intercambio de argumentos entre la ciudadanía y el Estado con posibilidades reales de influencia y participación social en los asuntos políticos. Por su parte, el gobierno electrónico se concibe como una versión más restringida, en términos administrativos y técnicos, del uso de internet y las plataformas de información y comunicación para la ejecución de las diferentes operaciones estatales.

\footnotetext{
${ }^{10}$ No es simple hacer una diferencia estricta de ambos conceptos; de hecho, en buena parte de la literatura y en programas de gobierno estatales, son utilizados indistintamente. Además, su definición llega a abarcar términos como e-participation, e-democracy, e-governance, entre otros. Este trabajo asume como diferencia sustancial entre ambas nociones la expuesta en términos históricos y de apertura efectiva del Gobierno.
} 
De este modo, el gobierno abierto busca que las propuestas y demandas que emergen de la sociedad civil se aprovechen como insumo para alimentar el proceso de toma y ejecución de decisiones políticas. Por lo que las interfaces mediáticas y electrónicas se convierten en un sistema real y efectivo para que el Estado atienda los inputs provenientes de la ciudadanía (Miller, 2009; Freeman, 2013). Valga añadir: para que el proceso sea efectivo y pueda materializarse, hace falta no solo que ingresen las demandas ciudadanas al sistema, sino que el Estado, con diferentes mecanismos institucionales, genere un feedback constante que facilite procesos de retroalimentación con la sociedad civil para optimizar, cambiar o corregir las decisiones y políticas públicas implementadas (Nath, 2011).

Es justo esta posibilidad de retroalimentación y reconocimiento de la ciudadanía como agente activo que aporta demandas y soluciones la que permite establecer conexiones directas con los tres principios de gobierno abierto defendidos por la Administration of Barack H. Obama (2009). Esta forma de gobierno reconoce que la información está ampliamente distribuida en la sociedad, que la transparencia promueve accountability, que la participación ciudadana hace al Estado mucho más eficaz en la implementación de soluciones y que la colaboración involucra diferentes sectores sociales que deben tenerse en cuenta para la ejecución de acciones público-administrativas encaminadas a favorecer a la sociedad civil (Ramírez-Alujas, 2012; Villoria, 2014). En definitiva, el gobierno abierto propugna la "horizontalización” de las relaciones entre Estado y sociedad (Villoria, 2014).

\section{Transparencia y accountability en la era digital}

Como hemos visto, los discursos sobre gobierno electrónico y gobierno abierto están íntimamente ligados a las nociones de transparencia y circulación libre y efectiva de la información. A mayor circulación de datos desde el Estado hacia la sociedad civil, se esperan mayores niveles de transparencia, pues tal proceso permitiría un mejor control ciudadano del comportamiento estatal (Dutton, 2009; Dawes, 2010; Concha y Naser, 2012). En esa lógica, la tecnología dispone las condiciones de comunicación entre el Gobierno y la ciudadanía. Es evidente que, sin información sobre las actuaciones estatales ni canales de interacción con las administraciones, los ciudadanos no podrían hacer seguimiento ni exigir cuentas a sus representantes. Por ello, los portales electrónicos de las entidades públicas son una buena forma de ilustrar medios para la transparencia y la interactividad.

Así pues, la transparencia es entendida como la voluntad de poner a disposición de los ciudadanos los detalles pormenorizados de la conducta administrativa, haciendo visibles sus acciones y decisiones con el propósito de mejorar los niveles de accountability, ya que la información revelada permite contrastar la veracidad de los propósitos o declaraciones estatales. Lo anterior conduce a un mayor monitoreo y control desde entes civiles como asambleas ciudadanas, organizaciones de derechos humanos o los mismos individuos (Keane, 2009). Al respecto, Freeman afirma que "las estructuras políticas 
tradicionales siguen siendo importantes, pero han aumentado el escrutinio y las demandas de control desde fuentes externas para transformar la posición central del Estado en la política” (las traducciones son nuestras) (2013, p. 355). La transparencia permite, además, alcanzar una gestión pública eficiente y eficaz, pues es insumo para los análisis y procesos de decisiones económicas. ${ }^{11}$

Y aunque el discurso sobre la transparencia no es nuevo, el uso de aplicaciones y software para la gestión, la consolidación y el análisis de grandes cantidades de información (big data) sí es novedoso. Con el uso generalizado de estas aplicaciones, se han logrado establecer plataformas para el intercambio de datos, la colaboración entre diferentes actores que facilitan la identificación de necesidades y el diseño de las soluciones (Dawes, 2010).

Ahora bien, ċcuál es el papel del accountability en relación con el valor de la transparencia defendido por el nuevo paradigma del gobierno abierto? Como habrá podido notarse, los límites entre ambas nociones no son muy claros y, en todo caso, el análisis del discurso formal del presidente Barack H. Obama sobre esta nueva empresa y las tesis producidas desde la academia dejan claro que ambos procesos están imbricados y se afectan mutuamente. Asegurar transparencia garantizará el accountability, en especial el accountability social, al permitir que haya una relación mucho más fuerte entre los ciudadanos y sus gobiernos.

\section{La materialización del ideal de gobierno abierto: usos y aplicaciones}

Pueden rastrearse numerosos portales web y aplicaciones alrededor del mundo que se han valido de las TIC para optimizar procesos político-administrativos. Por esa razón, este último apartado trata de dar cuenta, no de las experiencias pormenorizadas, sino de cuatro tipos de prácticas que permiten describir en sentido amplio el uso y la reutilización de datos en el gobierno abierto.

En el primer grupo de prácticas, se destacan los usos de corte meramente institucional, enfocados en trámites y prestación de servicios, ejercicios muy propios de lo que se ha caracterizado en la sección anterior como gobierno electrónico. Las experiencias que hacen parte de este primer conjunto buscan fortalecer y transformar los gobiernos locales y nacionales mediante el uso de las TIC, con miras a hacer más eficientes los procesos administrativos en el aparato estatal y lograr con ello una prestación más eficaz de servicios y trámites a la sociedad civil (Ramírez-Alujas, 2011). En ese sentido, las administraciones deben propender a la automatización y simplificación de todos sus

\footnotetext{
${ }^{11}$ Un ejemplo significativo de la importancia de la transparencia para generar mayor eficacia económica puede encontrarse en los estudios de Stiglitz $(2000,2002)$. En ellos, el autor muestra cómo la transparencia es un elemento fundamental para las economías, pues permite, por ejemplo, poner a disposición de potenciales inversionistas, productores y consumidores información relevante en cuanto al desarrollo y funcionamiento de la economía para la optimización de los procesos de toma de decisiones.
} 
trámites y oferta institucional con internet, dispositivos móviles y televisión digital. Aprovechando las ventajas que ofrecen los softwares para analizar, sistematizar y consolidar información, y capitalizando los recursos propios de la red en internet para consultar y compartir esa misma información, se hace un llamado desde el gobierno abierto para que se integren servicios y cadenas de trámites de diferentes dependencias estatales con un solo canal de información. ${ }^{12}$ Así las cosas, se habla de un Estado 100 \% en línea, disponible para el ciudadano sin limitaciones temporales o espaciales, lo cual hace posible además la construcción de indicadores de desempeño a partir del seguimiento a las actividades realizadas por funcionarios según instrumentos de gestión.

En la misma línea de la optimización de servicios gubernamentales a partir del uso de plataformas comunicacionales e informacionales, aparece como segundo conjunto de prácticas aquellas que son mucho más específicas y técnicas. Ejemplos de ello se hallan en Nath (2011), quien muestra cómo para el clima, la apertura de datos que antes de la década de 1970 era propiedad única y exclusiva de la Administración Nacional Oceánica y Atmosférica de los Estados Unidos, ha permitido que medios de comunicación, desarrolladores de aplicaciones móviles y organizaciones públicas y privadas puedan apropiarse de la información estatal para implementar sistemas de seguimiento y monitoreo sobre el clima con una gran cantidad de usos para los ciudadanos. ${ }^{13}$

El tercer conjunto de prácticas y desarrollo de aplicaciones a partir de la aparición del gobierno abierto tiene que ver con una manera particular e importante de utilización de datos abiertos por terceros (agremiaciones sociales, ciudadanos o empresas privadas). Según informes de la Comisión para la Explotación Comercial de la Información del Sector Público Europeo (2012) y el Estudio de caracterización del sector infomediario en España (Observatorio Nacional de las Telecomunicaciones y de la Sociedad de la Información, 2014), los recursos generados por la reutilización de datos públicos para promover el desarrollo de aplicaciones móviles utilizadas por los ciudadanos rebasa con creces los EUR 70 ooo millones para Europa y más de EUR 5000 millones para España. Más aún, un estudio revelado por la consultora McKinsey (Manyika, Chui, Farrell, Van Kuiken, Groves y Almasi, 2013) estima que la liberación y reutilización de datos abiertos por los Estados en el mundo

\footnotetext{
${ }^{12}$ El modelo adoptado por países como Colombia, Uruguay y Argentina ha sido el de crear ventanillas únicas, virtuales y físicas, en las cuales los ciudadanos pueden acercarse para consultar información sobre la oferta institucional, oportunidades de empleo, salud y educación, seguridad ciudadana, trámites fiscales y administrativos, entre muchos otros. Asimismo, el modelo descrito en este primer conjunto de aplicaciones busca que el ciudadano no deba aportar información que se supone está ya en manos del Estado, gracias a la interoperabilidad e integración de bases de datos. Estos adelantos han sido formalizados por el Decreto 2693/2012, de 21 de diciembre en Colombia, el Decreto 450/009/2009, de 8 de octubre en Uruguay, el Decreto 1172/2003, de 3 de diciembre y el Decreto 512/2009, de 7 de mayo en Argentina.

${ }^{13}$ El transporte es también un tema importante. En San Francisco, cerca del $20 \%$ de las aplicaciones desarrolladas tienen que ver con este tema. Para información detallada sobre las aplicaciones desarrolladas, puede verse https://datasf.org/
} 
podría generar como valor agregado, una suma que supera los USD 3 billones al año solo en sectores como educación, transporte, electricidad, gas y petróleo, salud y usos financieros.

Es difícil estimar para América Latina ${ }^{14}$ el valor económico aproximado que se genera a partir de la libre circulación de los datos gubernamentales, pero la aparición de leyes y decretos que buscan respaldar y asegurar el gobierno abierto en los Estados de la región y la promoción de experiencias para fomentar la reutilización de datos electrónicos desde la sociedad civil y la implementación de portales para publicar información estatal indican futuros avances con repercusiones políticas, económicas y sociales que sería interesante analizar desde la academia.

El último conjunto de prácticas y aplicaciones particulares de gobierno abierto tiene que ver con el uso de datos estatales para reforzar los vínculos entre el Estado y la sociedad civil, esto es, la entrega de conjuntos de datos a los ciudadanos y la creación de canales virtuales que propician la interacción entre los administradores de lo público y los individuos (RamírezAlujas, 2010, 2012). Las experiencias de este tipo son muy versátiles. Pueden encontrarse redes sociales para empleados y funcionarios de gobierno ${ }^{15}$ y plataformas para el monitoreo de los movimientos financieros del Estado. ${ }^{16}$ Los canales de intercambio entre la sociedad y el Estado en el gobierno abierto buscan, además, generar sistemas de inputs que permitan, no solo la consulta y el aprovechamiento de la apertura de datos por los sujetos, sino, además, que estos hagan parte de la gestión gubernamental en el diseño de soluciones a problemas sociales. En concordancia con la línea trazada por el cuarto grupo, un esfuerzo destacable en el continente americano es la Iniciativa Latinoamericana por los Datos Abiertos, red compuesta por diferentes organismos públicos y privados que busca conectar experiencias exitosas en el continente para el uso de datos abiertos en lo que tiene que ver con cualificación del conocimiento y promoción de la transparencia y el accountability. ${ }^{17}$

\section{Más allá del discurso sobre la transparencia: el papel de las TIC en la promoción del accountability}

Las ideas expuestas en el apartado anterior dan cuenta del potencial poder de las TIC para promover la participación ciudadana y el control político. No obstante, el

\footnotetext{
${ }^{14}$ Los estudios sobre el peso económico de la reutilización de datos públicos en América Latina son significativamente escasos cuando no inexistentes. Sin embargo, se conoce del uso de datos abiertos por empresas para desarrollar aplicaciones que permiten hacer seguimiento a los servicios de transporte de rutas escolares y ubicación en tiempo real de los menores por sus padres, ofrecer servicios a sectores campesinos y ganaderos sobre subastas comerciales para la compra de insumos, aplicaciones con fines educativos para conectar y gestionar dudas, entre otras. ${ }^{15}$ Una de las experiencias que mayor impacto ha tenido en este sentido es GovLoop, que puede consultarse en https://www.govloop.com

${ }^{16}$ La plataforma a la que se hace referencia es Foreign Assistance y puede consultarse en https://www.foreignassistance.gov/

${ }^{17}$ La información a propósito de esta iniciativa puede ampliarse en https://idatosabiertos.org/
} 
desarrollo de estas se encuentra en una etapa prematura; para el gobierno abierto y la transparencia, aún son limitados los alcances reales del accountability y la participación efectiva de los ciudadanos ${ }^{18}$ en el control del poder político.

Según lo expuesto, la transparencia se ha articulado como discurso en la última década al uso de las TIC con el fin de hacer visible el ejercicio estatal. ${ }^{19} \mathrm{El}$ derecho de los ciudadanos a estar informados es uno de los principios fundamentales de la democracia y del paradigma contemporáneo del gobierno abierto. Las agencias internacionales como la Organización para la Cooperación y el Desarrollo Económicos, el Banco Mundial, la Comisión Económica para América Latina y el Caribe y la Alianza para el Gobierno Abierto han defendido la importancia de la información y de la disponibilidad de los datos para alcanzar mayor control sobre el poder político y fomentar el empoderamiento y la participación ciudadana, haciendo explícito el vínculo existente entre transparencia y accountability.

Ahora, dicho vínculo no homologa los conceptos y menos las prácticas a las que dan lugar. Según Fox (2007), no pueden confundirse transparencia y accountability: de hecho, solo ciertas formas de transparencia podrían conducir a algún tipo de accountability. El académico suscribe dos tipos de transparencias:

- La transparencia opaca o borrosa que se refiere a la información diseminada o que no revela fielmente la manera en que las instituciones públicas actúan y a la divulgación de datos solo en términos nominales, que no resultan confiables o que exigen de los ciudadanos una gran inversión de tiempo y esfuerzo para sistematizarlos, analizarlos, ponderarlos y transformarlos en información útil y transparente.

- La transparencia clara que alude a políticas y programas de acceso a la información que revelan datos confiables, reutilizables, de acceso libre y en formatos estandarizados sobre el desempeño estatal (Fox, 2007).

Las dos formas de transparencia enunciadas son las que permiten encontrar las diferencias y relaciones con el accountability. Como vimos, este tiene dos dimensiones básicas: answerability y enforcement ${ }^{20}$ (Schedler, 1999, 2008; O’Donnell, 1998, 2004;

\footnotetext{
${ }^{18}$ Sobre este punto, a propósito de las primeras etapas de desarrollo del gobierno abierto, su énfasis en la transparencia y la dificultad para el desarrollo de aplicaciones que permitan hablar realmente de participación ciudadana vía internet, puede consultarse Ahn y Bretschneider (2011), Dutton (2009), Ramírez-Alujas (2010), Bekkers (2012), Freeman (2013); y a propósito del momento de la mayoría de los Estados como generadores solo de estrategias progobierno electrónico, Lee y Kwak (2011).

${ }^{19}$ Fox (2007) efectúa una crítica a la conceptualización reciente sobre la transparencia, afirmando que "ha recibido una atención mucho más práctica que conceptual" (p. 65). Sin embargo, sugiere que existen textos que hacen un mayor énfasis en la claridad teórica y conceptual (Florini, 2003; Graham, 2002; Hood y Heald, 2006, Monsiváis, 2005). ${ }^{20}$ Es válido aclarar, llegados a este punto, que el enforcement no necesariamente alude a la sanción punitiva, sino que puede remitir también a recompensas o estímulos por conductas sanas y que observen preceptos legales en el ejercicio público administrativo (Schedler, 2008; O’Donnell, 2004).
} 
Fox, 2007). Ante esta composición del accountability, la transparencia clara podría relacionarse solo con la dimensión del answerability, puesto que ambas impelen a las autoridades administrativas a informar y justificar sus acciones. En esa lógica, cuando se habla solo de acceso a la información, sin que sea clara la dimensión punitiva del control, es válido hablar de transparencia, pero en ningún sentido de accountability. La condición necesaria e irrestricta para que este pueda darse por completo exige la activación de agencias de control estatales que procedan, en efecto, con sanciones a los funcionarios públicos por omisiones o excesos en el ejercicio de sus funciones; la mera circulación de información resulta insuficiente.

Debe matizarse, pues, la tesis defendida por el paradigma de gobierno abierto según la cual una mayor apertura de datos y circulación de la información conduce a mayores niveles de accountability. Como se ha observado, la transparencia y apertura en términos informativos de la actuación estatal es condición necesaria, pero no suficiente para generar sistemas plenos de control. Por lo dicho, es preciso afirmar que los ideales de gobierno abierto distan aún de promover un ejercicio real de participación civil y control político, pues se acercan más a manifestaciones de gobierno que alcanzan a ser legitimadas por la sociedad gracias a que homologan el valor de la transparencia con la práctica del accountability.

De ahí la premura por adelantar reflexiones críticas frente a los ejercicios gubernamentales de transparencia, para establecer si promueven o no accountability, pero, además, la necesidad de seguir alimentando las iniciativas de control político que tienen germen en la sociedad civil, reconociendo el potencial que poseen las TIC.

\section{Las plataformas móviles como medio para la identificación de necesidades sociales}

El discurso sobre gobierno abierto y la teoría del accountability comparten dos características: 1) el afán de domesticar el poder político y 2) el deseo de menguar la brecha entre el Estado y los ciudadanos, con el fin de que las decisiones políticas respondan de forma adecuada a las necesidades sociales y las solucione de manera eficiente.

Las TIC aparecen como alternativa para canalizar demandas y necesidades colectivas hacia las estancias responsables de implementar soluciones o aplicar las sanciones a que dieran lugar. Un argumento a favor de esta tesis es propuesto por Freeman (2013). Según el investigador, las TIC pueden reflejar los marcos interpretativos y los intereses de los actores involucrados en el proceso de intercambio de información gracias a las posibilidades que brindan en la recepción, el almacenamiento y el análisis de grandes cantidades de datos a mayor velocidad y de manera más sofisticada. Se agilizan así diferentes procesos comunicativos entre la sociedad y el Estado, ya que las condiciones temporales o geográficas dejan de tener un peso relevante. 
Sin embargo, la atención a las demandas surgidas desde la ciudadanía y transmitidas con las redes electrónicas de información no son atendidas per se. Para ello, es fundamental que los administradores públicos tengan la voluntad de recibir y tramitar tales observaciones. Esto conduce a reconocer a los individuos y actores involucrados (organizaciones sociales y empresas privadas) como pares en los procesos de identificación de necesidades colectivas e implementación y evaluación de alternativas de solución (Bekkers, 2012; Ahn y Bretschneider, 2011). De tal forma que "horizontalizar" las relaciones entre el Estado y la sociedad civil permite dar el paso desde el discurso de la transparencia, dirigido en una sola vía desde los administradores públicos a la sociedad, a un proceso comunicativo que involucra múltiples actores para la resolución de necesidades. Así las cosas, son los mismos ciudadanos quienes orientarían las acciones de los funcionarios públicos, ejerciendo una suerte de control político antes de la ejecución de acciones o, lo que sería lo mismo, un accountability ex ante. ${ }^{21}$

Una de las alternativas más coherentes para materializar los ideales de control y transmisión de inquietudes desde la sociedad hacia los entes gubernamentales es propuesta a partir de la utilización de terminales móviles, ${ }^{22}$ en especial los celulares. Dichos dispositivos se adaptan de forma muy particular a las aspiraciones de consumo social de los individuos en la contemporaneidad, importando cada vez menos las diferencias de edad (Castells, 2001, 2009). Por ejemplo, McGrath (2011) muestra de manera práctica la facilidad que representa el uso de celulares conectados a internet para acceder a diferentes sitios en línea o comunicarse con otras personas por vías digitales gracias a la portabilidad y el abaratamiento de los costos que representan. ${ }^{23}$ En un contexto interconectado con múltiples plataformas y redes electrónicas, se encuentran oportunidades para saber en tiempo real la naturaleza y el origen de cualquier tipo de demandas o insatisfacciones ciudadanas.

De igual forma, será cada vez más viable la posibilidad de sistematizar y publicar en tiempo real los datos, las demandas o las evaluaciones al sistema estatal hechas por los ciudadanos desde sus terminales móviles en sitios destinados para ello (blogs,

\footnotetext{
${ }^{21}$ El término accountability ex ante, abordado especialmente en estudios económicos, sobre todo en aquellos que versan sobre política económica, por ejemplo Burki (2014) y Polishchuk (2014), es tomado por los autores de este trabajo con el fin de dar cuenta de un tipo de accountability que busca ejercer un control previo sobre aquellos que toman decisiones públicas y que se basa en que las necesidades ciudadanas sean realmente identificadas por el Estado. A diferencia de los tipos de accountability identificados a lo largo del artículo, el accountability ex ante no ejerce control sobre las acciones gubernamentales que ya han sido desplegadas, y a diferencia del answerability, no espera la justificación estatal sobre las medidas que buscan ejecutarse. Esta modalidad invierte la lógica comunicativa en la medida en que es el Estado el que, con diferentes medios, debería escuchar a la sociedad civil antes de emprender algún tipo de acción.

22 Por terminales móviles puede entenderse celulares, smartphones, phablets y tablets.

${ }_{23}$ McGrath (2011) da cuenta de cómo los computadores, por ejemplo, dejan de ser el dispositivo ideal en lo que tiene que ver con conexiones a internet para asuntos puntuales como mirar titulares de noticias, actualizar redes o intercambiar información. De hecho, siguiendo el reporte reseñado por Cisco (2017), para 2020 se estima que tres quintas partes del total de dispositivos conectados a la red serán dispositivos inteligentes como smartphones, phablets y tablets, esto es, el $67 \%$ de dispositivos, frente al $36 \%$ existentes en 2015.
} 
aplicativos o webs), lo cual permitiría que organizaciones sociales, centros de pensamiento y medios de comunicación contrasten el discurso y las acciones oficiales con los problemas reales identificados desde la ciudadanía. Lo anterior promovería una forma de control y presión de abajo hacia arriba sobre los representantes políticos, más en vía de ejecución o sugerencias de políticas que hacen falta desarrollar o implementar - accountability ex ante- que en lógica de monitorear los planes que ya se han emprendido desde las administraciones.

Según Freeman (2013), autores como Eggers (2005) y Damodaran, Olphert y Balatsoukas (2008) afirman que este tipo de participación ciudadana y abierta favorecería una mayor satisfacción de los individuos con el sistema público-administrativo y aumentaría la confianza en los representantes políticos. Tal tipo de participación asegura la correspondencia entre inversión de recursos y atención de necesidades mayoritarias, debido a que los procesos de toma de decisiones se tornan mucho más complejos respecto de la información gracias al ascenso, instancias institucionales, de flujos discursivos provenientes de esferas civiles que alimentan diagnósticos, la identificación de prioridades y destinación de esfuerzos respecto de la gestión e inversión social.

Así las cosas, estas propuestas enmarcadas dentro de una suerte de "gobernanza móvil" conducen a prácticas de gobierno abierto, en las cuales destacan ciudadanos activos que, conectados en red desde dispositivos socialmente masificados, pueden evaluar tanto el comportamiento de funcionarios como la evolución en la ejecución de planes administrativos y proponer soluciones a las necesidades propias de los territorios. Es necesario, si se quiere ser consecuente con el discurso sobre el gobierno abierto y obedecer a los principios de participación y colaboración que este promueve, que se creen aplicaciones móviles atractivas, desde agencias independientes y diferentes de las del Estado, que favorezcan y materialicen estos principios.

Ahora bien, una crítica adicional al discurso sobre el gobierno abierto se desprende de uno de sus principios fundamentales: lograr que los ciudadanos dispongan de conjuntos cualificados de información generada por los representantes y funcionarios públicos, para que ejerzan así funciones de monitoreo al desempeño estatal. Es difícil esperar que los individuos manifiesten un interés real en términos motivacionales y prácticos por descargar en sus computadores grandes sumas de datos agregados. ${ }^{24} \mathrm{El}$ hecho de que el Estado publique información no conduce directamente a que esta sea demandada y analizada por los sujetos (Marques y Pineda, 2014). De igual forma, se presentan problemas que tienen que ver con la confiabilidad y veracidad de aquello

\footnotetext{
${ }^{24}$ Teniendo en cuenta, además, para este punto, que estos datos, según los mismos principios del gobierno abierto, deben ser en bruto, es decir, no estar relacionados con variables ni filtrados. Esto se traduce en hojas de cálculo con bases de datos excesivamente amplias para la capacidad de análisis y seguimiento del ciudadano promedio.
} 
que se publica y con las deficiencias administrativas para actualizar los informes de las diferentes dependencias gubernamentales. ${ }^{25}$

No obstante, las organizaciones sociales, específicamente las ONG, los centros de pensamiento y, en alguna medida, los medios independientes de comunicación pueden devenir traductores, auditores y supervisores de la información generada por actores institucionales. Esto por una razón básica: sus labores de investigación, monitoreo y generación de análisis a propósito de la actuación de los gobiernos precisan de un insumo fundamental: la disponibilidad de los datos que promueve el discurso sobre gobierno abierto.

La premisa de sistematizar y traducir la información producida por el Estado a un lenguaje menos técnico para que pueda circular, en efecto, entre los ciudadanos, encuentra en las señaladas organizaciones civiles un depositario válido para el fortalecimiento de los procesos de accountability, porque son estas organizaciones las que cumplen cabalmente con el perfil requerido en términos personales y motivacionales, académicos y de capacidad instalada para emprender las tareas de depuración y análisis de los datos en bruto.

Asimismo, las organizaciones sociales y los centros de pensamiento pueden cualificar los procesos de ejecución de obras públicas y programas sociales con la autonomía y objetividad de sus análisis. Estas organizaciones encuentran en la disposición libre de datos e información insumos que las ayudan a reflexionar sobre sus objetos de estudio y así concretar su misión académica e investigativa. El producto de esa labor será, directa o indirectamente, una fuente para el análisis y seguimiento a políticas públicas gubernamentales.

La dinámica descrita propone reorientar tanto la cadena de transmisión de la información como de los actores que hacen parte del mismo proceso. Las organizaciones son, si se quiere, un filtro entre los datos estatales publicados y la ciudadanía o los individuos como actores particulares. Esta reorientación permite verificar la veracidad de los informes y la coherencia respecto de las agendas programáticas propuestas por los representantes políticos, al tiempo que favorece la entrega a la ciudadanía de información clara y de fácil acceso que integra variables de análisis mucho más comprehensivas y relacionadas con grupos sociales, dependencias estatales o necesidades territoriales concretas. La observación y el seguimiento constante por parte de especialistas a temas particulares permitirá activar las funciones de las agencias estatales de balance y asignadas, responsables de emprender procesos sancionatorios en el accountability ${ }^{26}$

\footnotetext{
${ }^{25}$ Sobre estas críticas y la manera en que muchas veces los procesos de datos abiertos terminan por ser más una herramienta legitimadora de los gobiernos que un insumo válido para un control político real desde la ciudadanía, puede revisarse Bechtold y Perrig (2014), Bekkers (2012) y Wong y Welch (2004).

${ }^{26}$ Es muy importante resaltar, frente a este aspecto, la existencia de múltiples agencias y organizaciones públicas, privadas, académicas e independientes que, haciendo uso de los datos abiertos y las TIC, alimentan los procesos de análisis y presentación de la información a la ciudadanía, promoviendo formas sociales de accountability. Estas comprenden amplios temas, desde organizaciones en Norteamérica que velan por la defensa de los derechos de poblaciones afro y latinos a partir de la generación de informes sobre la actuación de quienes dicen representar sus intereses: Color of Change, Voto Latino, hasta observatorios parlamentarios en Colombia
} 
en los casos en que ello llegara a ser necesario. Así que no son ya las TIC el único objeto sobre el que deba dirigirse la atención, sino más bien sobre las posibilidades que ellas brindan, en el contexto actual, para fortalecer los mecanismos de control político y rendición de cuentas y, en la misma medida, la propia democracia.

\section{Consideraciones finales}

$\mathrm{El}$ análisis de los discursos y las propuestas que buscan ligar el uso de herramientas digitales a la promoción de la transparencia y el control político permite identificar avances significativos en lo que concierne al desarrollo de aplicaciones y portales para la liberación de datos estatales. Sin embargo, no es muy clara la distinción entre las apuestas por la circulación libre de la información y las posibilidades reales de sanción y control sobre el Estado que ellas promueven. Esa falta de claridad permite formular desde la teoría del accountability alternativas para conciliar el anhelo de gobiernos abiertos y transparentes con herramientas de fiscalización, monitoreo y sanción a la conducta de representantes y funcionarios públicos.

Asimismo, el ideal del gobierno abierto por crear canales óptimos que promuevan la participación ciudadana en la supervisión a las acciones estatales y en el desarrollo de programas y proyectos gubernamentales se encuentra aún a medio camino. La gran mayoría de los usos identificados para internet en el seno de las administraciones públicas actuales se enmarca en el paradigma que hemos descrito como gobierno electrónico. Por tanto, adquiere relevancia pensar en instrumentos que permitan potenciar la incidencia de la sociedad civil sobre el Estado.

Es allí donde las plataformas móviles pueden hacer posible que los ciudadanos -agremiados o no-sumen demandas a propósito de fallas u omisiones en la implementación de políticas públicas o programas de gobierno que habrán de ser tramitadas por entes estatales y así materializar la presunción de Hernández y Arciniegas (2011) del accountability social como dispositivo naturalmente relacional, o de Peruzzotti cuando afirma que "la verticalidad de la accountability social tiene, tendenciosa e intencionalmente, un destino horizontalizado" (2010, p. 10).

De igual forma, se alerta sobre la premisa de reorientar la cadena de transmisión de la información que propone el gobierno abierto, con el fin de que la liberación de datos estatales tenga como público objetivo las agencias especializadas, las organizaciones sociales y los medios de comunicación. Estos agentes tienen la vocación de convertir estos datos en insumos prácticos y atractivos que la ciudadanía puede asimilar. De suerte que,

como Congreso Visible y sus capítulos regionales que buscan hacer seguimiento a legisladores durante sus periodos como representantes, suministrando insumos constantes fáciles de asimilar para la sociedad civil y que contribuyen tanto a los procesos de seguimiento a representantes políticos como a la cualificación de la toma de decisiones por parte de los ciudadanos en eventos electorales. 
de manera similar a como el accountability horizontal y vertical deben tender a complementarse para desplegar toda su fuerza (O’Donnell, 1998, 2004), el accountability social también deviene agente que facilita la interacción armónica con sus demás tipologías.

El control político ejercido por los individuos, las organizaciones sociales y los centros de pensamiento revitaliza la esfera público-deliberativa y la emergencia de formas de acción que, aunque focalizadas, dan cuenta de la preocupación por asuntos públicos que conducen a la generación de espacios de interrelación vía deliberación y comunicación (Hernández y Flórez, 2011). Todos estos actores permitirían materializar la forma de accountability ex ante: ninguna acción estatal será más transparente y coherente que aquella que parta del reconocimiento mismo de las necesidades identificadas y sistematizadas por los ciudadanos.

Finalmente, el panorama esbozado deja claro que es preciso ampliar las preguntas, en especial, en los sistemas políticos latinoamericanos alrededor de los potenciales usos de los datos abiertos. Hecho que implica reflexiones que tienen que ver con el papel activo del Estado frente a la voluntad de atraer organizaciones sociales y centros de pensamiento que capitalicen los insumos informacionales. De igual modo, debe analizarse qué tipo de avances legislativos son precisos para impulsar la seguridad informática y la reutilización de datos abiertos con fines económicos, ya que se ha visto que el manejo de estos puede crear grandes oportunidades de negocio y proyectarse como un factor cada vez más importante en la economía global. Aprovechar la información generada por los ciudadanos y el Estado, posible de sistematizar y analizar gracias a desarrollos en software y big data, abre las puertas a sistemas de control al gobierno previos y posteriores mucho más depurados que repercuten no solo en la salud del sistema democrático, sino también en la revitalización de la sociedad civil y la creación de nuevos renglones económicos.

\section{Referencias}

Administration of Barack H. Obama (2009). Memorandum on Transparenc $y$ and Open Government. Recuperado de https://www.archives.gov/files/cui/ documents/2009-WH-memo-on-transparency-and-open-government.pdf

Ahn, M. J. y Bretschneider, S. (2011). Politics of e-government: e-government and the political control of bureaucracy. Public Administration Review, 71(3), 414-424.

Bechtold, S. y Perrig, A. (2014). Accountability in future internet architectures. Communications of the ACM, 57(9), 21-23.

Bekkers, V. (2012). Why does e-government looks as it does? Looking beyond the explanatory emptiness of the e-government concept. Information Polity: The International Journal of Government \& Democracy in the Information Age, $17(3 / 4), 329-342$. 
Bovens, M. (2007). Analysing and assessing accountability: A conceptual framework. European Law Journal, 13(4), 447-468.

Burki, S. J. (2014, mayo 19). Ex-ante accountability. En The Express Tribune. Recuperado de https://tribune.com.pk/story/710043/ex-ante-accountability/

Castells, M. (2001). La era de la información: el poder de la identidad. México: Siglo XXI.

Castells, M. (2009). Comunicación y poder. Madrid: Alianza Editorial.

Cisco (2017, marzo 28). Cisco Visual Networking Index: Global Mobile Data Traffic Forecast Update, 2015-2020 White Paper. Recuperado de http://cisco.com/c/ en/us/solutions/collateral/service-provider/visual-networking-index-vni/ mobile-white-paper-c11-520862.html

Concha, G. y Naser, A. (eds.) (2012). El desafío hacia el gobierno abierto en la hora de la igualdad. Santiago de Chile: Naciones Unidas/Comisión Económica para América Latina y el Caribe.

Dahl, R. (1999). La democracia: una guía para los ciudadanos. Madrid: Taurus.

Damodaran, L., Olphert, W. y Balatsoukas, P. (2008). Democratizing local e-government: The role of virtual dialogue. En T. Janowski y T. A. Pardo (eds.), Proceedings of the 2nd international conference on theory and practice of electronic governance (ICEGOV'o8) (pp. 388-393). Nueva York: ACM.

Dawes, S. S. (2010). Stewardship and usefulness: Policy principles for information-based transparency. Government Information Quarterly, 27(4), 377-383.

Decreto 1172/2003, de 3 de diciembre, acceso a la información pública.

Decreto 512/2009, de 5 de mayo, créase el Grupo de Trabajo Multisectorial, que tendrá por finalidad concertar e impulsar la "Estrategia de Agenda Digital de la República Argentina”.

Decreto 450/2009, 8 de octubre, principios y lóneas estratégicas para el gobierno en red.

Decreto 2693/2012, de 21 de diciembre, por el cual se establecen los lineamientos generales de la Estrategia de Gobierno en Línea de la República de Colombia, se reglamentan parcialmente las Leyes 1341 de 2009y 1450 de 2011, y se dictan otras disposiciones.

Directiva 2013/37/UE del Parlamento Europeo y del Consejo, de 26 de junio de 2013 , por la que se modifica la Directiva 2003/98/CE relativa a la reutilización de la información del sector público Texto pertinente a efectos del EEE.

Dunn, J. (1999). Situating democratic political accountability. En A. Przeworski, S. C. Stokes y B. Manin (eds.), Democracy, accountability, and representation (pp. 329-345). Cambridge: Cambridge University Press. 
Dutton, W. H. (2009). The fifth estate emerging through the network of networks. Prometheus, 27(1), 1-15.

Eggers, W. D. (2005). Government 2.o: Using technology to improve education, cut red tape, reduce gridlock, and enhance democracy. Lanham, Maryland: Rowman \& Littlefield Publishers Inc.

Elster, J. (1999). Accountability in Athenian politics. En A. Przeworski, S. C. Stokes y B. Manin (eds.), Democracy, accountability, and representation (pp. 253-278). Cambridge: Cambridge University Press.

Florini, A. (2003). The coming democracy: New rules for running a new world. Washington DC: Island Press.

Fox, J. (2000). Civil society and political accountability: Propositions for discussion. Ponencia presentada en Democratic Accountability in Latin America, Kellogg Institute for International Studies, University of Notre Dame, Indiana.

Fox, J. (2007). The uncertain relationship between transparency and accountability. Development in Practice, 17(4-5), 663-671.

Freeman, J. (2013). E-government in the context of monitory democracy: Public participation and democratic reform. Media Asia, 4O(4), 352-362.

Graham, M. (2002). Democracy by disclosure: The rise of technopopulism. Washington DC: Brookings Institution.

Grau-Solés, M., Íñiguez-Rueda, L. y Subirats, J. (2011). ¿Cómo gobernar la complejidad? Invitación a una gobernanza urbana híbrida y relacional. Athenea Digital, 11(1), 63-84.

Halachmi, A. y Holzer, M. (2010). Citizen participation and performance measurement: Operationalizing democracy through better accountability. Public Administration Quarterly, 34(3), 378-399.

Hernández Quiñones, A. (2006). Modelos de democracia liberal representativa: limitaciones y promesas incumplidas. Co-Herencia, 3(4), 37-75.

Hernández Quiñones, A. y Arciniegas Muñoz, E. (2011). Aproximación conceptual. El accountability desde una perspectiva comparada en América Latina: estudios de caso. En A. Hernández Quiñones y E. Arciniegas Muñoz (comps.), Experiencias de accountability horizontal y social en América Latina: estudios de caso comparados en São Paulo, México D. F., Caracas y Bogotá (pp. 20-46). Bogotá: Universidad de los Andes.

Hernández Quiñones, A. y Flórez Herrera, J. (2011). Análisis y estudio de experiencias de accountability social en América Latina. Bogotá: Universidad de los Andes. 
Hood, C. y Heald, D. (eds.) (2006). Transparency: The key to better governance? Oxford: British Academy/Oxford University Press.

Keane, J. (2009). The life and death of democracy. Londres: Simon \& Schuster.

Ke, W. y Wei, K. K. (2004). Successful e-government in Singapore. Communications of the ACM, 47(6), 95-99.

Kenney, C. D. (2000). Reflections on horizontal accountability: Democratic legitimacy, majority parties and democratic stability in Latin America. Ponencia presentada en The Conference on Institutions, Accountability, and Democratic Governance in Latin America, Kellogg Institute for International Studies, University of Notre Dame, Indiana.

Lee, G.-J. (2008). Study on group behavior in the cyberspace: Surrounding the candlelight vigil against Korea-U.S. beef import agreement.

Lee, G. y Kwak, Y. (2011). An open government implementation model: Moving to increased public engagement. Washington DC: IBM Center for the Business of Government.

Lee, S. M., Tan, X. y Trimi, S. (2005). Current practices of leading e-government countries. Communications of the ACM, 48(10), 99-104.

Locke, J. (2004). Segundo tratado sobre el gobierno civil. Madrid: Alianza Editorial.

López Muñoz, L. (coord.) (2014). Informe del sector TICy los contenidos en España 2013. Madrid: Observatorio Nacional de las Telecomunicaciones y de la Sociedad de la Información. Recuperado de http://www.ontsi.red.es/ontsi/sites/ontsi/files/ informe_del_sector_tic_y_los_contenidos_en_espana_2013_edicion_2014.pdf

Manyika, J., Chui, M., Farrell, D., Kuiken, S. van, Groves, P. y Almasi Doshi, E. (2013). Open data: Unlocking innovation and performance with liquid information. McKinsey Global Institute. Recuperado de https:// www.mckinsey.com/business-functions/digital-mckinsey/our-insights/ open-data-unlocking-innovation-and-performance-with-liquid-information

Marques, M. y Pineda, C (2014). Desafíos y limitaciones en el proceso de datos abiertos de salud y sanidad: lecciones a partir de las experiencias de Brasil y España. Ponencia presentada en el XIX Congreso Internacional del CLAD sobre la Reforma del Estado y de la Administración Pública, Quito, Ecuador.

McGrath, M. (2011). Technology, media, and political participation. National Civic Review, 10o(3), 41-44. 
Miller, L. (2009). E-petitions at Westminster: The way forward for democracy? Parliamentary Affairs, 62(1), 162-177.

Monsivaís, A. (comp.) (2005). Políticas de transparencia: ciudadanía y rendición de cuentas. México: Instituto Federal de Acceso a la Información Pública/Centro Mexicano para la Filantropía.

Montoya Londoño, C. (2010). Alianzas entre medios de comunicación y organizaciones de la sociedad civil: balances y propuestas para fortalecer la democracia y los derechos humanos en América Latina. Bogotá: Pontificia Universidad Javeriana.

Nath, J. (2011). Reimagining government in the digital age. National Civic Review, $100(3), 19-23$.

O'Donnell, G. A. (1998). Horizontal accountability in new democracies. Journal of Democracy, 9(3), 112-126.

O'Donnell, G. A. (2001). Acerca de varias accountabilities y sus interrelaciones. En E. Peruzzotti y C. Smulovitz (eds.), Controlando la política: ciudadanos y medios en las nuevas democracias latinoamericanas (pp. 87-102). Buenos Aires: Temas.

O’Donnell, G. A. (2004). Accountability horizontal: la institucionalización legal de la desconfianza política. Revista Española de Ciencia Política, 11, 11-31.

Organización para la Cooperación y el Desarrollo Económico (2003). Promise and problems of e-democracy: Challenges of online citizen engagement. París: Organización para la Cooperación y el Desarrollo Económico. Recuperado de http://www.oecd.org/governance/digital-government/35176328.pdf

Peruzzotti, E. (2008). Marco conceptual de la rendición de cuentas. Ponencia presentada en la Reunión Técnica de la Comisión de Rendición de Cuentas de la Organización Centroamericana y del Caribe de Entidades de Fiscalización Superior, Auditoría General de la Nación, Buenos Aires, Argentina.

Peruzzotti, E. (2010). Entidades de fiscalización superior y participación ciudadana. Ponencia presentada en la Reunión Técnica de la Comisión de Rendición de Cuentas de la Organización Centroamericana y del Caribe de Entidades de Fiscalización Superior, Auditoría General de la Nación, Buenos Aires, Argentina.

Peruzzotti, E. (2012). Broadening the notion of democratic accountability: Participatory innovation in Latin America. Polity, 44(4), 625-642.

Peruzzotti, E. y Smulovitz, C. (2002). Accountability social: la otra cara del control. En E. Peruzzotti y C. Smulovitz (eds.), Controlando la política: ciudadanos y medios en las democracias latinoamericanas (pp. 1-38). Buenos Aires: Temas. 
Polishchuk, L. (2014). External agency: Accountability ex-ante [Video]. Recuperado de https://www.coursera.org/learn/public-economics/lecture/nuOYw /5-2-external-agency-accountability-ex-ante

Ramírez-Alujas, Á. (2010). Innovación en la gestión pública y open government (gobierno abierto): una vieja nueva idea. Revista Buen Gobierno, 9, 94-133.

Ramírez-Alujas, Á. V. (2011). Gobierno abierto y modernización de la gestión pública: tendencias actuales y el (inevitable) camino que viene: reflexiones seminales. Revista Enfoques: Ciencia Política y Administración Pública, 9(15), 99-125.

Ramírez-Alujas, Á. V. (2012). Gobierno abierto es la respuesta: ¿cuál era la pregunta? Más Poder Local, 12, 14-22.

Ríos Ramírez, A., Cortés Arbeláez, A., Suárez Valencia, M. C. y Fuentes Vélez, L. (2014). Accountability: aproximación conceptual desde la filosofía política y la ciencia política. Colombia Internacional, 82, 261-288.

Ríos Ramírez, A. y Trujillo Urrea, J. P. (2014). Accountability societal: herramienta de gobernanza contra la corrupción. En A. Eslava (ed.), Oro como fortuna: instituciones, capital social y gobernanza de la minería aurífera colombiana (pp. 363-408). Medellín: Universidad Eafit.

Schedler, A. (1999). Conceptualizing accountability. En A. Schedler, L. Diamond y M. Plattner (eds.), The self-restraining state: Power and accountability in new democracies (pp. 13-28). Colorado: Lynne Rienner.

Schedler, A. (2008). ¿Qué es la rendición de cuentas? México: Instituto Federal de Acceso a la Información Pública.

Sosa, J. (2011). Transparencia y rendición de cuentas: un ensayo introductorio. En J. Sosa (ed.), Transparencia y rendición de cuentas. México: Siglo XXI.

Stiglitz, J. (2000). The contribution of the economics of information to the twentieth century economics. The Quarterly Journal of Economics, 115(4), 1441-1478.

Stiglitz, J. (2002). Transparency in government. En R. Islam (ed.) The right to tell: The role of mass media in economic development (pp. 27-45). Washington DC: The World Bank.

Thompson, J. (2001). El escándalo político: poder y visibilidad en la era de los medios de comunicación. Barcelona: Paidós.

Villoria, M. (2012). Transparencia y gobierno abierto: ¿qué gobierno quiere el gobierno abierto? Ponencia presentada en el XVII Congreso Internacional del CLAD sobre la Reforma del Estado y de la Administración Pública, Cartagena, Colombia. 
West, D. M. (2004). E-government and the transformation of service delivery and citizen attitudes. Public Administration Review, 64(1), 15-27.

Wong, W. y Welch, E. (2004). Does e-government promote accountability? A comparative analysis of website openness and government accountability. Governance, $17(2), 275^{-297}$ 
